\title{
Integrability of a disordered Heisenberg spin-1/2 chain
}

\author{
L. F. Santo: * $^{-1}$ \\ Department of Physics and Astronomy, Michigan State University, East Lansing, MI 48824
}

\begin{abstract}
We investigate how the transition from integrability to nonintegrability occurs by changing the parameters of the Hamiltonian of a Heisenberg spin-1/2 chain with defects. Randomly distributed defects may lead to quantum chaos. A similar behavior is obtained in the presence of a single defect out of the edges of the chain, suggesting that randomness is not the cause of chaos in these systems, but the mere presence of a defect.

PACS numbers: 75.10.Jm, 05.50.+q, 05.45.Mt
\end{abstract}

\section{INTRODUCTION}

Random matrix theory has long been used to describe the spectra of complex systems, such as nuclei, molecules, and mesoscopic solids [1]. Recently it has been used in the study of strongly correlated spin systems [2, [3]. The statistical properties of the quantum energy spectrum are strongly influenced by the underlying classical dynamics. The level spacing distribution of a classical integrable system is Poissonian, $P_{P}(s)=\exp (-s)$, while the level statistics of a chaotic system is given by the WignerDyson distribution, $P_{W D}(s)=(\pi s / 2) \exp \left(-\pi s^{2} / 4\right)$. The Wigner-Dyson distribution is obtained in random matrix theory and it reproduces the level repulsion of chaotic dynamics. These two distributions characterize, respectively, the localized and the metallic phase in the Anderson model of disordered systems. At the critical point between the two phases an intermediate level spacing statistics occurs [4].

The problem of localization and the statistical properties of the spectra for the case of just one particle has long been understood, but only recently has the problem of many-body systems been addressed [2, 3]. When more than one particle is present in the system, the interaction between them has to be taken into account. The interplay between interaction and disorder is a challenging problem in today's condensed matter physics and it can lead to new and unexpected effects [5].

Here, we consider a one-dimensional Heisenberg spin$1 / 2$ chain with defects and several excitations. A defect corresponds to the site where the energy splitting is different from all the others. It is obtained by applying a different magnetic field in the $z$ direction to the chosen site. In the absence of defects this homogeneous system is integrable and is solved with the Bethe ansatz []. $]$. Its level distribution is therefore Poissonian. As random onsite magnetic fields are turned on and their mean-square amplitude starts increasing, the system undergoes a transition and becomes chaotic. But by further increasing the mean-square amplitude, localization eventually takes place and the distribution becomes Poissonian again. We

*Electronic address: santos@pa.msu.edu determine the crossover from integrability to quantum chaos in such disordered spin chain. In addition, we discuss that the cause for nonintegrability is not the randomness of the system, but the mere presence of defects. If only one defect is placed out of the edges of the chain and if the defect excess energy is of the order of the interaction strength, the system is also chaotic. The same sort of transition integrable-chaotic-integrable is obtained as the defect excess energy increases.

We consider only nearest neighbor interaction. The Hamiltonian describing the system is

$$
H=\sum_{n=1}^{L}\left(h_{n}-\frac{J}{2} \delta_{n, 1}-\frac{J}{2} \delta_{n, L}\right) \sigma_{n}^{z}+\sum_{n}^{L-1} \frac{J}{4} \vec{\sigma}_{n} \cdot \vec{\sigma}_{n+1},
$$

where $\hbar=1$ and $\vec{\sigma}$ are Pauli matrices. There are $L$ sites. Each site $n$ is subjected to a magnetic field in the $z$ direction, giving the energy splitting $h_{n}$. The chain is ideal whenever all sites have the same energy splitting. A defect corresponds to the site whose energy splitting differs from the others.

For simplicity, we work with an isotropic chain, that is, the coupling constant $J$ for the diagonal Ising interaction $\sigma_{n}^{z} \sigma_{n+1}^{z}$ is equal to the coupling constant for the $X Y$-type interaction $\sigma_{n}^{x} \sigma_{n+1}^{x}+\sigma_{n}^{y} \sigma_{n+1}^{y}$. This last term is responsible for propagating the excitation through the chain. A single-particle excitation corresponds to a spin pointing up.

In this model, the $z$ component of the total spin $\sum_{n=1}^{L} S_{n}^{z}$ is conserved, so states with different number of excitations are not coupled. We therefore look at the level spacing distributions for sectors with the same number of excitations. Since we are interested in determining if the system is integrable or chaotic, we focus on the sector with the largest number of states, that is the sector with $L / 2$ excitations. This is the region where chaos should set in first.

In a very large system the boundary conditions have no effects, but numerical calculations are limited to a finite number of sites. In a periodic (or closed) chain we found too many degenerate states, so we decided to work with a chain with free boundaries (or open chain). Both systems, closed or open, are known to be integrable in the absence of defects. They are solved with the Bethe ansatz method [6]. An open chain with defects only on 
the edges is also integrable [7]. Here we choose an open chain with defects of values $-J / 2$ on the edges. Such values nullify the border effects.

We work with $L=12$ sites and 6 excitations, which gives us 924 states. A matrix of such size, $924 \times 924$, is sufficient to have good statistics, as illustrated in Fig. 1] In both plots we have the Poisson distribution (dot-dashed line) and the Wigner-Dyson distribution (long-dashed line). The spacings $s$ correspond to $S / M$, where $M$ is the mean level spacing and $S$ is the actual spacing. Both histograms are normalized to 1 . The histogram at the top of the figure shows that the level spacings of the eigenvalues of a diagonal random matrix of such size is well described by the Poisson distribution. The one at the bottom of the figure shows that the level spacings of the eigenvalues of a random matrix of this size agrees very well with the Wigner-Dyson distribution. The random elements have a Gaussian distribution.

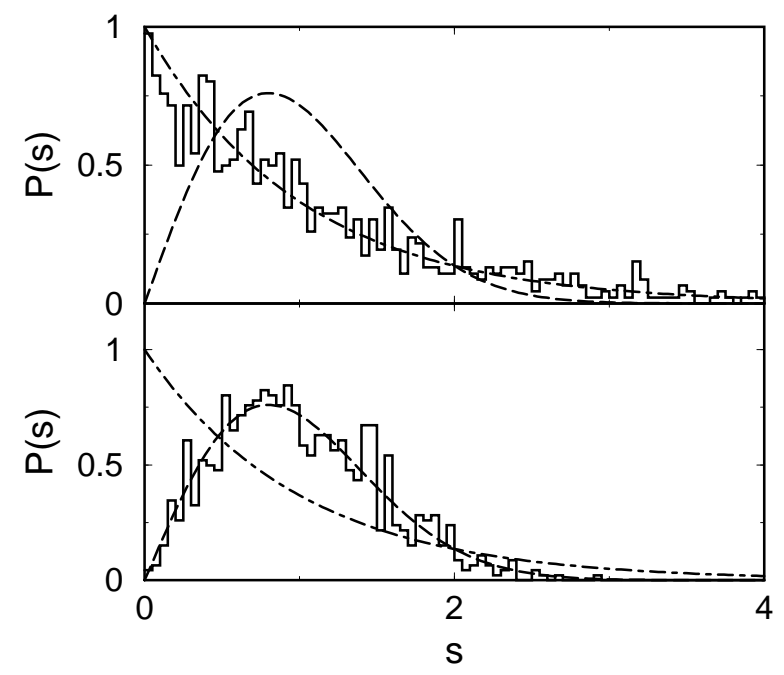

FIG. 1: In both graphs dot-dashed line gives the Poisson distribution and long-dashed line corresponds to the WignerDyson distribution. The histogram at the top gives the level spacing distribution for a diagonal random matrix of dimension $924 \times 924$. At the bottom it gives the level distribution for a random matrix of this size.

\section{RANDOMLY DISTRIBUTED DEFECTS}

First we analyze the case of random magnetic fields along the $z$ direction. The energy splitting of each site is given by $h_{n}=h+d_{n}$, where $d_{n}$ 's are uncorrelated random numbers with a Gaussian distribution: $\left\langle d_{n}\right\rangle=0$ and $\left\langle d_{n} d_{m}\right\rangle=d^{2} \delta_{n, m}$. When $d=0$ the system is integrable and a Poisson distribution is obtained, as the histogram at the top of Fig. 2 shows. As $d$ increases the system undergoes a transition and becomes chaotic, the WignerDyson distribution is obtained, as can be seen from the histogram in the middle of Fig. 2 However, as we further increase $d$ and it becomes much larger than $J$, the system becomes localized. As expected, a Poisson distribution reappears (see the bottom of Fig. 2). Large $d$ corresponds to a random diagonal matrix with negligible off diagonal elements.
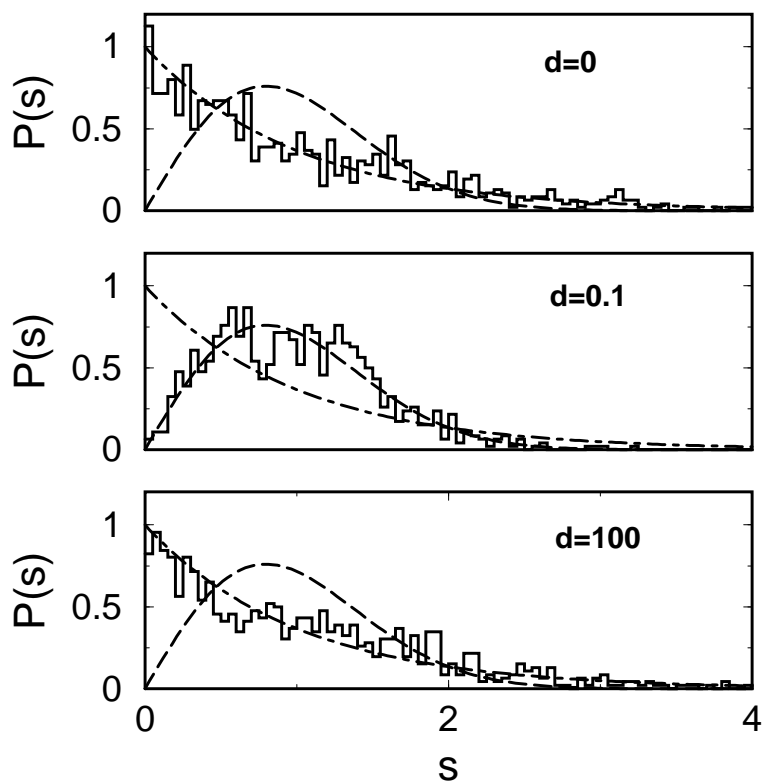

FIG. 2: The histograms correspond to the actual level distribution for random on-site magnetic fields. We choose $J=1$. Dot-dashed line gives the Poisson distribution and long-dashed line corresponds to the Wigner-Dyson distribution.

A more convenient way to analyze the evolution of the level spacing distributions with respect to the ratio $d / J$ is by using the parameter $\eta=\int_{0}^{s_{0}}[P(s)-$ $\left.P_{W D}(s)\right] d s / \int_{0}^{s_{0}}\left[P_{P}(s)-P_{W D}(s)\right] d s$, where $s_{0}=0.4729 \ldots$ is the intersection point of $P_{P}(s)$ and $P_{W D}[3,8]$. A regular system has $\eta=1$ and a chaotic system has $\eta=0$. The stars on Fig. 3] show the dependence of $\eta$ on $d / J$. There the transition integrable-chaotic-integrable is clear. The system is initially integrable, reaches its maximum chaoticity when $d \sim 10^{-1} J$ and then becomes integrable again as $d$ becomes much larger than $J$. At this last step, the energy splitting of each site becomes very different from all the others and the excitations become localized.

Besides considering the site energies as random numbers, another way to introduce disorder is by taking the non-diagonal (hopping) matrix elements at random. We assume that the off diagonal elements also have a Gaussian distribution and the mean square is given by $J_{r}$. Contrary to the case discussed before, random coupling (circles on Fig. 3) leads to chaos even when the energy splittings of all sites are the same $(d=0)$. Maximum chaoticity is again reached at $d \sim 10^{-1} J_{r}$, but there is now just one transition, from chaos to integrability. Such transition takes a little longer to happen than in the pre- 


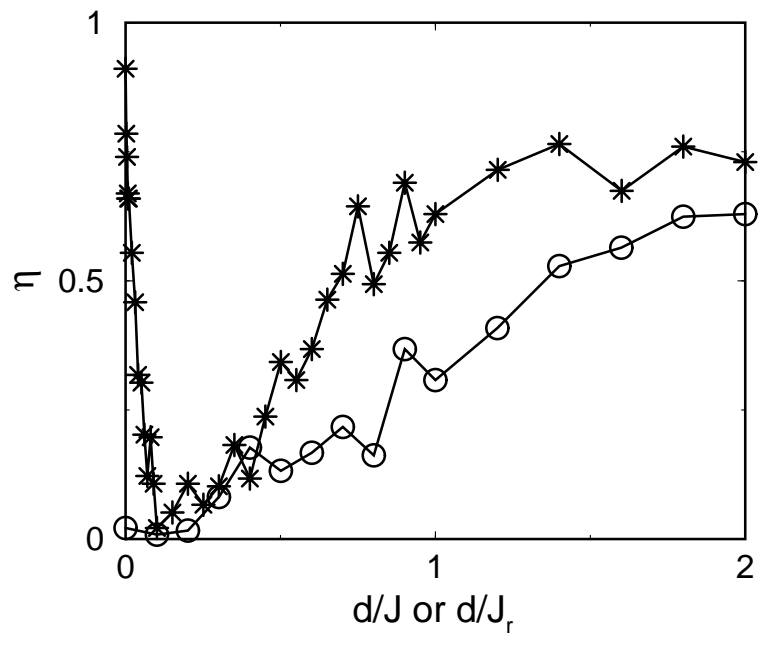

FIG. 3: Stars give the dependence of $\eta$ on the ratio $d / J$ when the on-site magnetic fields are random. Circles give the dependence of $\eta$ on the ratio $d / J_{r}$ when both diagonal and nondiagonal elements are random.

vious case, though localization is also attained once the ratio $d / J_{r}$ becomes large.

\section{ONE SINGLE DEFECT}

In the system treated here, chaos can be associated with randomness only when the coupling is random. In the case of a constant interaction strength, what is really responsible for the nonintegrability of the system is not the randomness of the energy splittings, but the mere existence of a defect. Let us consider again a constant coupling. The top of Fig. 固 shows that a Wigner-Dyson distribution can also be obtained when there is only one defect in the middle of the chain and the defect excess energy is of the order of the interaction strength. For this histogram all sites have the same energy splitting $h_{n}=h$, except site 6 , which has $h_{6}=h+J$.

Here too we use the parameter $\eta$ to study the evolution of the level spacing distribution with respect to the ratio $d_{6} / J$. The bottom of Fig. 团indicates a transitional behavior very similar to the case of constant coupling and random diagonal elements. The one-defect system is initially regular, but by increasing $d_{6}$ it becomes chaotic. The highest degree of chaoticity happens when $d_{6} \sim J$. The level repulsion now settles in more slowly than in the previous situation of random magnetic fields. As $d_{6}$ is further increased, an excitation on site 6 will become sitelocalized. This means that when the ratio $d_{6} / J$ becomes very large there are two kinds of states in the system: states with a localized excitation on site 6 and states with no excitation on the defect. These two types of states are not coupled. This system becomes equivalent to two smaller and uncoupled ideal chains, and integrability is therefore recovered.
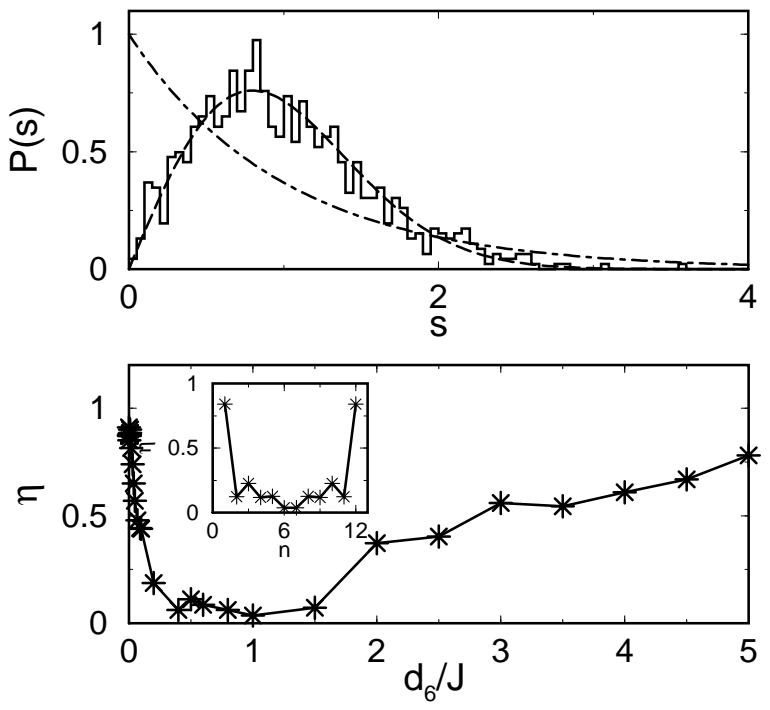

FIG. 4: Top panel: The histogram corresponds to the level distribution of the Heisenberg spin-1/2 chain with a defect on site 6 . The defect excess energy is equal to the interaction strength $J$. Dot-dashed and long-dashed lines give the Poisson and the Wigner-Dyson distributions respectively. Bottom panel: Dependence of the parameter $\eta$ on the ratio $d_{6} / J$. The defect is placed on site 6 . The inset gives the dependence of $\eta$ on the position of the defect; the defect excess energy is $J$.

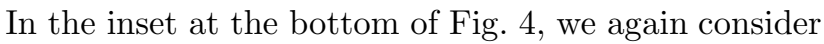
one single defect, whose excess energy is equal to $J$. It shows how the parameter $\eta$ depends on the position of the defect and confirms that the Heisenberg spin-1/2 system is integrable when it has defects placed on its edges.

\section{CONCLUSION}

We have shown that in a Heisenberg spin- $1 / 2$ chain, randomly distributed defects lead to the transition integrable-chaotic-integrable, according to the ratio $d / J$. When random off diagonal elements are also considered, there is only one transition from non-integrability to integrability. The transition integrable-chaotic-integrable is also observed when the coupling is constant and there is only one defect out of the edges of the chain. The level spacing distribution obtained in this case depends on how large the defect excess energy is in relation to the interaction strength.

Obtaining analytical solutions for disordered spin chains is not an easy task and in many cases is simply impossible. The algebraic version of the Bethe ansatz is often used to construct integrable Hamiltonians [9]. The analysis of level spacing distributions should therefore be useful to identify which real or constructed Hamiltonians are indeed integrable.

Understanding under what conditions disordered spin systems become integrable is not just relevant for condensed matter physics, but also for quantum computa- 
tion, since these systems are commonly used to model different proposals of quantum computers (see Ref. [5] and references therein).

\section{Acknowledgments}

We acknowledge support by the NSF through grant No. ITR-0085922 and would also like to thank C. O.
Escobar for helpful discussions.
[1] T. Guhr, A. Müller-Groeling, and H.A. Weidenmüller, Phys. Rep. 299, 190 (1998); E.P. Wigner, Ann. Phys. (N.Y.) 53, 36 (1951); M.L. Mehta, Random Matrices and the Statistical Theory of Energy Level (Academic Press, New York, 1967); B.L. Altshuler and B. I. Shklovski, Zh. Eksp. Teor. Fiz. 91, 220 (1986) [Sov. Phys. JETP 64, 127 (1985).]

[2] G. Montambaux, D. Poilblanc, J. Bellissard, and C. Sire, Phys. Rev. Lett. 70, 497 (1993); T. C. Hsu and J. C. Anglès d'Auriac, Phys. Rev. B 47, 14291 (1993); Y. Avishai, J. Richert, and R. Berkovits, Phys. Rev B 66, 052416 (2002).

[3] B. Georgeot and D.L. Shepelyansky, Phys. Rev. Lett. 81, 5129 (1998).

[4] B.I. Shklovskii, B. Shapiro, B.R. Sears, P. Lambrianides, and H.B. Shore, Phys. Rev. B 47, 11487 (1993); D. Braun,
G. Montambaux, and M. Pascaud, Phys. Rev. Lett. 81, 1062 (1998).

[5] L.F. Santos and M.I. Dykman, e-print quant-ph/0303130 M.I. Dykman and L.F.Santos, e-print cond-mat/0308026 (to appear in J. Phys. A).

[6] H. A. Bethe, Z. Phys. 71, 205 (1931); C. N. Yang and C. P. Yang, Phys. Rev. 150, 321, 327 (1966); F. C. Alcaraz, M. N. Barber, and M. T. Batchelor, Ann. of Phys. 182, 280 (1988).

[7] F. C. Alcaraz, M. N. Barber, M. T. Batchelor, R. J. Baxter, and G. R. W. Quispel, J. Phys. A 20, 6397 (1987).

[8] P. Jacquod and D. L. Shepelyansky, Phys. Rev. Lett. 79, 1837 (1997).

[9] A. A. Zvyagin, J. Phys. A: Math. Gen. 34, R21 (2001). 